\title{
Effect of Stimulation Polarity of Transcranial Direct Current Stimulation on Non-dominant Hand Function
}

\author{
Min Kyun Sohn, M.D., Bong Ok Kim, M.D., Hyun Tak Song, M.D.
}

Department of Rehabilitation Medicine, Chungnam National University School of Medicine, Daejeon 301-172, Korea

\begin{abstract}
Objective To evaluate motor excitability and hand function on the non-dominant side according to the polarity of transcranial direct current stimulation (tDCS) on the motor cortex in a healthy person.

Method tDCS was applied to the hand motor cortex for 15 minutes at an intensity of $1 \mathrm{~mA}$ in 28 healthy righthanded adults. Subjects were divided randomly into four groups: an anodal tDCS of the non-dominant hemisphere group, a cathodal tDCS of the non-dominant hemisphere group, an anodal tDCS of the dominant hemisphere group, and a sham group. We measured the motor evoked potential (MEP) in the abductor pollicis brevis and Jabsen-Taylor hand function test (JTT) in the non-dominant hand prior to and following tDCS. All study procedures were done under double-blind design.

Results There was a significant increase in the MEP amplitude and a significant improvement in the JTT in the non-dominant hand following anodal tDCS of the non-dominant hemisphere $(\mathrm{p}<0.05)$. But there was no change in JTT and a significant decrease in the MEP amplitude in the non-dominant hand following cathodal tDCS on the non-dominant hemisphere and anodal tDCS of the dominant hemisphere.

Conclusion Non-dominant hand function is improved by increased excitability of the motor cortex. Although motor cortex excitability is decreased in a healthy person, non-dominant hand function is maintained. A homeostatic mechanism in the brain might therefore be involved in preserving this function. Further studies are warranted to examine brain functions to clarify this mechanism.
\end{abstract}

Key Words tDCS, Transcranial direct current stimulation, Jabsen-Taylor hand function test, Cortical excitability

Received December 6, 2010; Accepted November 2, 2011

Corresponding author: Hyun Tak Song

Department of Rehabilitation Medicine, Chungnam National University Hospital, 282, Munhwa-ro, Jung-gu, Daejeon 301-172, Korea

Tel: +82-42-280-7811 2, Fax: +82-42-256-6056, E-mail: smfahen@cnuh. co.kr

(c) This is an open-access article distributed under the terms of the Creative Commons Attribution Non-Commercial License (http:// creativecommons.org/licenses/by-nc/3.0) which permits unrestricted noncommercial use, distribution, and reproduction in any medium, provided the original work is properly cited.

Copyright () 2012 by Korean Academy of Rehabilitation Medicine

\section{INTRODUCTION}

As described by Priori et al., ${ }^{1}$ non-invasive stimulation of the brain using transcranial direct current stimulation (tDCS) can modulate the activity of the cerebral cortex with the application of electrical current to the scalp, and may induce brain plasticity. The effects of tDCS are variable depending on the location and polarity of the electrode. Anodal stimulation increases the excitability of the cerebral cortex whereas cathodal stimulation 
decreases it. ${ }^{2,3}$ It has been reported that motor evoked potentials (MEP) are increased ${ }^{2-4}$ and the functions of the contralateral hand are increased when the primary motor area is stimulated with anodal tDCS in healthy adults. ${ }^{5,6}$ It has also been reported that MEP is increased ${ }^{7,8}$ and the functions of the affected hand are significantly improved if anodal tDCS is applied to the affected primary motor area in stroke patients. ${ }^{7-10}$ Furthermore, cathodal tDCS of the primary motor cortex in healthy adults decreases the contralateral MEP amplitude by the reduction of cortical excitability. $^{2,3}$

The modulation of the excitability of the cerebral hemisphere causes indirect changes in the excitability of the contralateral cerebral hemisphere through the interhemispheric projection. Therefore, if the tDCS altered the excitability in a specific area of the brain, it would cause indirect changes in the excitability of the corresponding area on the contralateral side. ${ }^{11,12}$ Because the major mechanism of interhemispheric interaction is inhibition, decreased excitability of one side of the primary motor area will increase excitability of the contralateral side. ${ }^{13}$ Inhibition of primary motor cortex by cathodal tDCS activates the contralateral motor cortex and leads to increased ipsilateral hand functions. ${ }^{14}$ In addition, the affected hand function was improved by the application of cathodal tDCS to the non-affected primary motor area in stroke patients. ${ }^{15-17}$ An increased excitability on one side leads to a decreased excitability on the other side. Therefore, it can be predicted that contradictory results will occur to hand function between the ipsilateral and the contralateral side. Most studies have reported improved contralateral hand functions by increased excitability or improved ipsilateral hand function by decreased excitability of motor cortex. Vines et al. ${ }^{14}$ reported that anodal stimulation of the dominant hemisphere increased dominant hand function approximately $10 \%$ and decreased non-dominant hand function less than $2 \%$. However the relationship between changes in hand functions and cortical excitability could not be identified because the MEP was not measured.

Boggio et al. ${ }^{6}$ demonstrated that anodal tDCS of the dominant hemisphere or the non-dominant hemisphere in healthy adults caused significant changes in nondominant hand function through evaluation of the Jabsen-Taylor hand function test (JTT). However, there were no significant changes in dominant hand function.
This implies that dominant hand function was not improved due to a difficulty in further increasing the excitability of the dominant hemisphere following anodal tDCS application because it was already maximally activated. In the non-dominant hemisphere, however, the functional changes occurred due to the decreased use of the non-dominant hand. Accordingly, increased excitability of the non-dominant hemisphere by anodal tDCS would reverse the above changes to some extent and then lead to increases in non-dominant hand functions.

Little is known about the correlations between changes in the MEP and those in hand functions following stimulation of the cerebral cortex. Given the above background, we attempted to examine the correlations between the degree of activity of the cerebral cortex and functional changes following application of tDCS in healthy adults. We also attempted to determine the effects of changes in cerebral cortex activity on hand function in patients with brain injury such as stroke.

\section{MATERIALS AND METHODS}

\section{Subjects}

The current study was investigated 28 healthy righthanded adults ( 15 men and 13 women) with a mean age of 22.6 \pm 1.2 years. Inclusion criteria included a lack of a past history of diseases such as seizure or brain disease and that of other medical or surgical history. Subjects were fully informed of the side effects and risks that they might experience during participation in the current study. Then, subjects submitted a written informed consent. Subjects were simply randomized to four groups with seven subjects each. The current study was conducted under a double-blind design and a maximal number of subjects were selected for Friedmann's test. In the NDA (non-dominant hemisphere anodal stimulation) group, including three men and four women, the mean age was $22.1 \pm 2.2$ years. In the NDC (non-dominant hemisphere cathodal stimulation) group, consisting of four men and three women, the mean age was $21.3 \pm 1.2$ years. In the $S$ (sham stimulation) group, composed of four men and three women, the mean age was $22.0 \pm 2.1$ years. In the DA (dominant hemisphere anodal stimulation) group, containing four men and three women, the mean age was $21.7 \pm 1.5$ years. There 
Table 1. Comparisons of Age, Sex, and Pre-stimulation Measures between the Four Groups and Changes of MEP Amplitude and Jabsen-Taylor Test (JTT) Times after tDCS of Motor Cortex

\begin{tabular}{|c|c|c|c|c|c|c|}
\hline & \multirow{2}{*}{$\begin{array}{c}\text { Age } \\
\text { (years) }\end{array}$} & \multirow{2}{*}{$\begin{array}{c}\text { Sex } \\
\text { (male : female) }\end{array}$} & \multicolumn{2}{|c|}{ MEP amplitude (mV) } & \multicolumn{2}{|c|}{ JTT time (sec) } \\
\hline & & & Pre & Post & Pre & Post \\
\hline NDA & $22.1 \pm 2.2$ & $3: 4$ & $1.80 \pm 0.5$ & $2.78 \pm 0.6^{*}$ & $45.75 \pm 2.1$ & $41.07 \pm 2.0^{*}$ \\
\hline NDC & $21.3 \pm 1.2$ & $4: 3$ & $2.58 \pm 0.9$ & $1.23 \pm 0.6^{*}$ & $43.26 \pm 4.5$ & $42.43 \pm 5.2$ \\
\hline DA & $21.7 \pm 1.5$ & $4: 3$ & $2.15 \pm 0.5$ & $1.21 \pm 0.4^{*}$ & $43.01 \pm 3.5$ & $43.27 \pm 3.2$ \\
\hline S & $22.0 \pm 2.1$ & $4: 3$ & $2.23 \pm 0.2$ & $2.11 \pm 0.5$ & $43.23 \pm 5.5$ & $43.45 \pm 4.9$ \\
\hline
\end{tabular}

Values given are mean \pm standard deviation, ${ }^{*} \mathrm{p}<0.05$

NDA: Non-dominant anodal stimulation group, NDC: Non-dominant cathodal stimulation group, DA: Dominant anodal stimulation group, S: Sham stimulation group

was no significant difference in age or male-to-female ratio between the four groups (Table 1).

\section{Methods}

Transcranial direct current stimulation (tDCS): tDCS was given to the primary motor cortex (M1) at a magnitude of $1 \mathrm{~mA}$ for $15 \mathrm{~min}$ using a $5 \times 5 \mathrm{~cm}$ sponge electrode with the use of Phoressor II Auto instrument (Iomed, Salt Lake City, USA) with the subjects in a sitting position. The non-dominant hemisphere was stimulated in the NDA group, the NDC group, and the S group. The nondominant primary motor cortex was stimulated with the anode in the NDA group, and the cathode in the NDC group. The reference electrode was placed on the contralateral supraorbital region. Although an electrode was placed in the S group similarly to the NDA group, the electrical stimulation was given at a magnitude of $1 \mathrm{~mA}$ only for 30 seconds initially. In the DA group, the anode was placed in the primary motor area of the dominant hemisphere. Prior to and following tDCS, MEP and JTT were measured.

Motor evoked potential (MEP) test: MEP was measured by stimulating the cerebral hemisphere using a 70-mm, figure 8-shaped coil with a Magpro ${ }^{\circledR}$ X100 instrument (Medtronic Inc, farum, Denmark). Subjects underwent the test in a sitting position on a chair with both arms completely relaxed. With regard to the site of the stimulation of the primary motor cortex, we selected a spot where the maximal amplitude was induced from the contralateral abductor pollicis brevis (APB). Using the stimulator, resting motor threshold was assessed as the lowest intensity able to evoke a MEP of more than 50 $\mu \mathrm{V}$ in at least five out of ten consecutive trials. Then, we performed a stimulation at an intensity corresponding to $120 \%$ of resting motor threshold. ${ }^{11}$ We measured the latency and amplitude of the MEP from the APB. To minimize time-dependent changes in the location of the magnetic stimulation and that of the recording site, the site of stimulation was marked at a $1-\mathrm{cm}$ gap over a rubber cap with the use of a ruler. As the location of the recording site, the area where the electrode was attached was marked using a marker.

Jabsen-Taylor hand function test (JTT): The JTT has been universally used to evaluate hand functions in patients with impaired functions or coordination of hand. This was done not only because each item of this test reflects proficient functions of the hand and arms that are needed to do daily activities, but also, because those functions can be compared within the normal range for which validity and reliability has been demonstrated. ${ }^{6,18,19}$ In the current study, as described earlier, we performed a JTT for the left hand, the non-dominant side. First, to minimize the degree of the reduction of time depending on the times of participation, we priory performed the JTT six times. This was based on previous reports that all subjects showed no further time shortening based on their experience in taking the test at a mean frequency of six times prior to their participation in the experimental study. ${ }^{6}$ The JTT is composed of seven items including writing, turning over a card, holding a small object and then placing it in a can, placing a small object in a can with the use of a spoon, building a block, moving a light can and moving a heavy can. The time to complete each item was measured and then the times for all items were summed.

Statistical analysis: Statistical analysis was performed using SPSS 12.0. Two-way repeated measures ANOVA was used to evaluate the effects of $\operatorname{TIME}_{\text {(base, post) }}$ and 
INTERVENTION $_{(\mathrm{NDA}, \mathrm{NDC}, \mathrm{S}, \mathrm{DA})}$ on MEP latency and amplitude and total JTT time. Post hoc analysis was performed using Student-Newman-Keuls (SNK) methods.

To compare changes between prior to and following the tDCS, percentile values of the amplitude of the MEP and total JTT times were calculated. Between group comparisons were done using one-way ANOVA for the difference between INTERVENTION ${ }_{(\mathrm{NDA}, \mathrm{NDC}, \mathrm{S}, \mathrm{DA})}$. Pearson correlation analysis was done to identify the strength of correlations. Statistical significance was set at $\mathrm{p}<0.05$.

\section{RESULTS}

\section{Motor evoked potentials test}

There was no significant difference in MEP latency of the non-dominant hand between the four groups prior to the test, and no significant change after tDCS application in all groups. Also, there was no significant difference in MEP amplitude between the four groups $\left(\mathrm{F}_{3,24}=2.269\right.$, $\mathrm{p}=0.106$ ) prior to the test, but there were significant changes following $\left(\mathrm{F}_{1,24}=16.405, \mathrm{p}<0.001\right)$ and significant interactions $\left(\mathrm{F}_{3,24}=34.809, \mathrm{p}<0.001\right)$ between the types of stimulation and changes after stimulation. In post hoc tests, the MEP amplitude was significant increased in the NDA group and significantly decreased in both the NDC and the DA group (SNK: p<0.05) (Table 1) (Fig. 1).

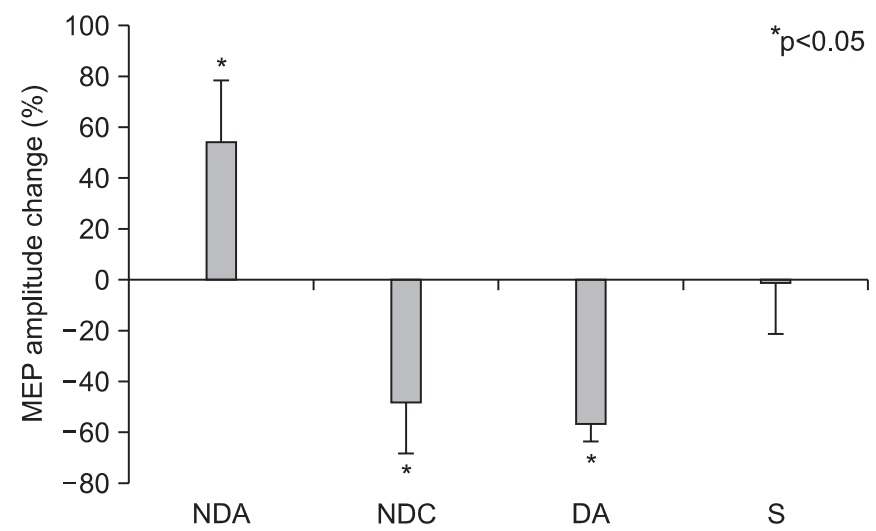

Fig. 1. The MEP amplitude in the non-dominant abductor pollicis brevis before and after tDCS of the primary motor cortex showed a significant increase in the non-dominant anodal stimulation group (NDA), and a decrease in the non-dominant cathodal stimulation group (NDC) and the dominant anodal stimulation group (DA). Values are mean \pm standard deviation. Sham stimulation group(s).

\section{Jabsen-Taylor hand function test}

Prior to the stimulation, there was no significant difference in total JTT time between the four groups $\left(F_{3,24}=0.0292, p=0.993\right)$. However, there was a significant change in JTT time after stimulation $\left(\mathrm{F}_{1,24}=23.852\right.$, $\mathrm{p}<0.001)$ and a significant interaction between the types of stimulation and JTT time $\left(\mathrm{F}_{3,24}=20.942, \mathrm{p}<0.001\right)$. Post hoc tests showed that there was no significant change in the NDC group, the DA group and the S group. In the NDA group, however, the total time lapsed was significantly shortened (SNK: p<0.05) (Fig. 2, 3) (Table 1).

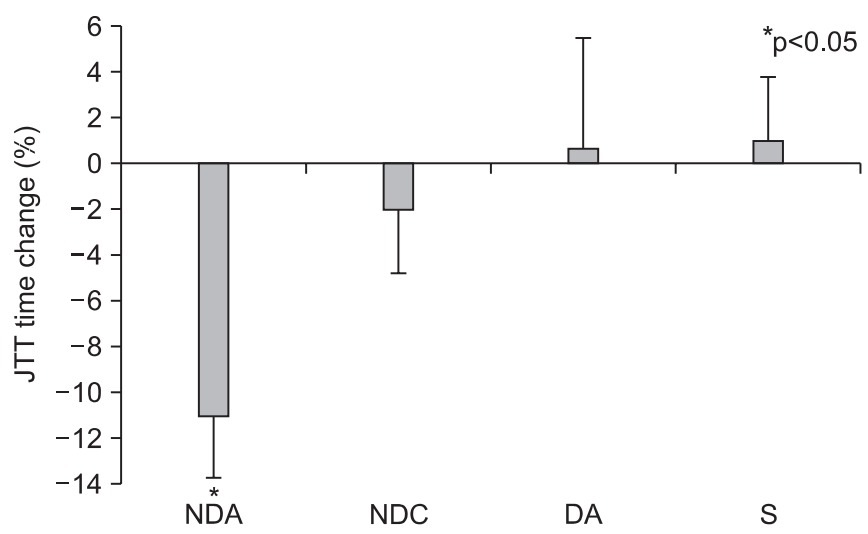

Fig. 2. The total time of the Jabsen-Taylor test (JTT) in the non-dominant hand before and after tDCS of the primary motor cortex showed a significant decrease in the nondominant anodal stimulation group (NDA). Values are mean \pm standard deviation. Sham stimulation group(s).

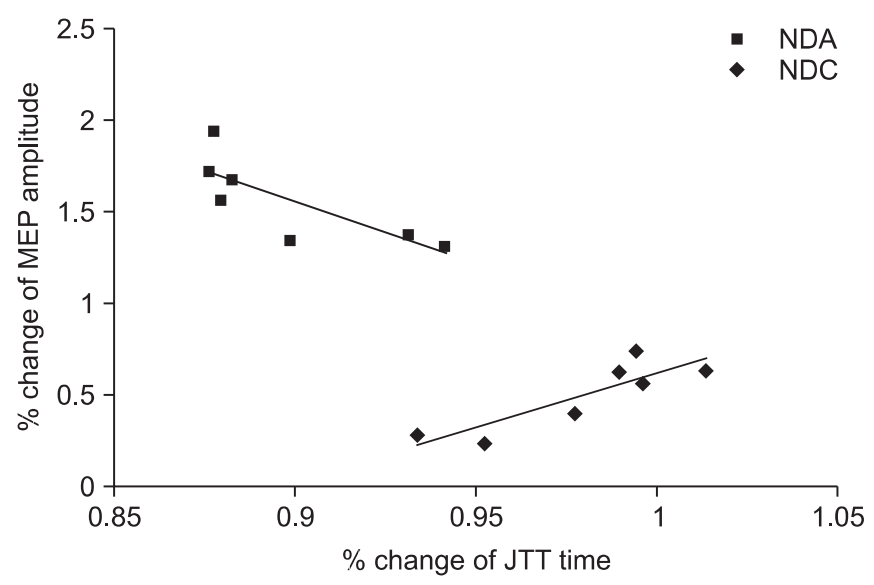

Fig. 3. There was a negative correlation between changes in percentile value of the MEP amplitude and Jabsen-Taylor test (JTT) in the non-dominant anodal stimulation group (NDA) $(\mathrm{r}=-0.843, \mathrm{p}<0.05)$ and a positive correlation in the non-dominant cathodal stimulation group (NDC) $(\mathrm{r}=0.878$, $\mathrm{p}<0.01)$. 


\section{Correlation between the two tests}

Analysis of the correlations between the percentile changes in MEP amplitude and JTT time after tDCS application showed a significant negative correlation in the NDA group (correlation coefficient $=-0.843, \mathrm{p}=0.017$ ), and a significant positive correlation in the NDC group (correlation coefficient $=0.878, \mathrm{p}=0.009$ ). In the DA group and the $S$ group, there was no significant correlation (Fig. $3)$.

\section{DISCUSSION}

In the current study, following anodal tDCS of the cerebral cortex, the MEP amplitude was increased and the JTT time was decreased on the contralateral side. Following contralateral cathodal tDCS or ipsilateral tDCS of the cerebral cortex, the MEP amplitude was significantly decreased, but there was no significant change in hand function. In other words, although cortical excitability of the non-dominant hemisphere was decreased by direct cathodal stimulation or indirect contralateral anodal stimulation, there was a lack of evidence of impaired functions in the non-dominant hand.

Boggio et al..$^{6,16}$ and Hummel et al. ${ }^{20}$ performed anodal tDCS at an intensity of $1 \mathrm{~mA}$ for 20 minutes and reported that JTT time was significantly decreased of $8.9 \%$ and $9.4 \%$, respectively. These values were close to the $10.3 \%$ seen in our current study. These results indicate that the nervous tissue of the primary motor area was depolarized, and this led to increased activity of the corticospinal tract following the use of tDCS. Consequently, increased activity of the motor tract directly induced improvement of arm functions. ${ }^{8}$ Jang et al. ${ }^{21}$ reported that the activity of the cerebral cortex was increased on fMRI following anodal tDCS. We also demonstrated increases in MEP amplitude following the use of anodal tDCS. This implies that improved motor functions following brain stimulation might arise from increased activity of the primary motor cortex.

On the other hand, Ko et al. ${ }^{8}$ performed upper limb function tests through a box-and-block test, grip power and lateral prehension power tests, and reported that there was a significant positive correlation between the degree of improvement of functions and increases in the MEP amplitude. Also, in the current study, according to Pearson correlation analysis, there was a significant negative correlation between the percentile values of the changes in MEP amplitude and those in JTT times following anodal stimulation of the non-dominant hemisphere.

Lang et al. ${ }^{3}$ reported that the MEP amplitude was decreased by $27 \%$ following cathodal stimulation. Our results showed similar findings. They reported, regardless whether the anodal or cathodal stimulation was given, there was no significant change in the MEP amplitude in the contralateral hemisphere. However, we had contradictory results. We found that the MEP amplitude on the contralateral side was decreased following anodal tDCS. The above authors explained that an interhemispheric connection had a higher level of threshold as compared with the corticospinal tract and this had no effect on the cerebral cortex on the contralateral side. In the current study, however, subjects were stimulated at an intensity of $1 \mathrm{~mA}$ in the same manner as used by the above authors. It has also been reported that the functions of the upper extremities on the affected side were improved following stimulation of the non-affected cerebral hemisphere at an intensity of 1 $\mathrm{mA}$ in patients with stroke. ${ }^{15,16}$ Further studies are needed to identify this mechanism.

Boggio et al.6 reported that there was no significant difference in the JTT in the sham group and the ipsilateral anodal stimulation group. These findings are in agreement with our results.

According to Vines et al., ${ }^{14}$ functions of the dominant hand were improved but functions of the non-dominant hand were impaired following anodal stimulation of the dominant hemisphere in normal right-handed adults. It was noted that the functions of the dominant hand were decreased but those of the non-dominant hand were improved following cathodal stimulation of the dominant hemisphere. These reports are contradictory to our results because non-dominant hand function was actually impaired in their study. According to the above authors, however, the functions of the non-dominant hand were improved by more than $10 \%$ following cathodal stimulation of the dominant hemisphere but those were decreased by less than $2 \%$ following anodal stimulation. Therefore, these results indicate that the actual degree of decrease in hand function was negligible. Vines et al. ${ }^{22}$ reported that there were changes in function 
of the dominant hand and the non-dominant hand following stimulation of the dominant hemisphere, but there was no significant change in the functions of the dominant hand even following stimulation of the nondominant hemisphere. Thus, these findings were thought to originate from asymmetry of the cerebral hemispheres. Although the above authors did not measure the activity of the cerebral cortex, because there was a response in the non-dominant hand under the same conditions of the stimulation, it can be inferred that there might be effects on the activity of the cerebral cortex. It can therefore be inferred that there might be an inconsistency between changes in the activity of the cerebral cortex and the functions of the hand. From this perspective, the above reports can be interpreted as being consistent with our results.

In the current study, the activity of the cerebral cortex of the non-dominant hemisphere was decreased to $47.7 \%$ and $56.3 \%$, respectively, following use of cathodal tDCS of the dominant hemisphere and anodal tDCS of the non-dominant hemisphere. But this did not lead to an actual decrease in the functions of the dominant hand. Hence, it has been speculated that another mechanism might be involved in maintaining hand functions in the human body. We used JTT, and Vines et al..$^{14,22}$ measured the sequential movement of fingers. Hence, we could not completely rule out effects due differences between their tests and ours.

To date, however, an insufficient number of studies have been conducted to examine correlations between changes in activity of the cerebral cortex and changes in hand functions. There are also an insufficient number of studies that have directly evaluated changes in the activity of the cerebral cortex on such imaging studies as fMRI. These deserve further study.

The current experimental study was performed in healthy right-handed adults, which was based on the subjective judgment of the authors. No objective evaluations were attempted to determine which hand was dominant. This is one of the limitations of the current study. However, because the current experimental study was performed for the left hand, a non-dominant hand of the subjects, we expected that there would not be any changes in the activity of the cerebral cortex or the functions of the hand following electrical stimulation.

\section{CONCLUSION}

MEP amplitude and the JTT for the non-dominant hand were measured after tDCS application at intensity of 1 $\mathrm{mA}$ for 15 minutes in healthy adults. Following anodal stimulation of the non-dominant hemisphere, there was a significant increase in the MEP amplitude and an improvement in the JTT. However, following anodal stimulation of the non-dominant hemisphere or cathodal stimulation of the dominant hemisphere, the MEP amplitude was significantly decreased, but there were no significant JTT changes. We presume that there is no consistent correlation between changes in the amplitude of the MEP and hand function, and a broad-spectrum of imaging studies such as fMRI might be needed to elucidate this mechanism. In addition, if our results are applied to patients with stroke, we would expect that the affected hand functions might be improved based on interhemispheric competition without impairments in non-affected hand function following anodal stimulation of the affected hemisphere or cathodal stimulation of the non-affected hemisphere.

\section{REFERENCES}

1. Priori A, Berardelli A, Rona S, Accornero N, Manfredi M. Polarization of the human motor cortex through the scalp. Neuroreport 1998; 9: 2257-2260

2. Nitsche MA, Paulus W. Excitability changes induced in the human motor cortex by weak transcranial direct current stimulation. J Physiol 2000; 527: 633-639

3. Lang N, Nitsche MA, Paulus W, Rothwell JC, Lemon RN. Effects of transcranial direct current stimulation over the human motor cortex on corticospinal and transcallosal excitability. Exp Brain Res 2004; 156: 439-443

4. Nitsche MA, Liebetanz D, Antal A, Lang N, Tergau F, Paulus W, Modulation of cortical excitability by weak direct current stimulation-technical, safety and functional aspects. Suppl Clin Neurophysiol 2003; 56: 255-276

5. Nitsche MA, Schauenburg A, Lang N, Liebetanz D, Exner C, Paulus W, Tergau F. Facilitation of implicit motor learning by weak transcranial direct current stimulation of the primary motor cortex in the human. J Cogn Neurosci 2003; 15: 619-626 
6. Boggio PS, Castro LO, Savagim EA, Bratie R, Cruz VC, Rocha RR, Rigonatti SP, Silva MT, Fregni F. Enhancement of non-dominant hand motor function by anodal transcranial direct current stimulation. Neurosci Lett 2006; 404: 232-236

7. Hummel F, Celnik P, Giraux P, Floel A, Wu WH, Gerloff C, Cohen LG. Effects of non-invasive cortical stimulation on skilled motor function in chronic stroke. Brain 2005; 128: 490-499

8. Ko MH, Han SH, Park SH, Seo JH. Improvement of hand function with transcranial direct current brain polarization in stroke patients. J Korean Acad Rehab Med 2009; 33: 259-264

9. Hesse S, Werner C, Schonhardt EM, Bardeleben A, Jenrich W, Kirker SG. Combined transcranial direct current stimulation and robot-assisted arm training in subacute stroke patients: a pilot study. Restor Nurol Neurosci 2007; 25: 9-15

10. Kim DY, Park CI, Jung KJ, Ohn SH, Park KD, Park JB, Oh YT. Improvement of chronic post-stroke hemiparetic upper limb function after 2 weeks transcranial direct current stimulation. J Korean Acad Rehab Med 2009; 33: 5-11

11. Gilio F, Rizzo V, Siebner HR, Rothwell JC. Effects on the right motor hand-area excitability produced by low-frequency rTMS over human contralateral homologous cortex. J Physiol 20003; 551; 563-573

12. Williams JA, Pascual-Leone A, Fregni F. Interhemispheric modulation induced by cortical stimulation and motor training. Phys Ther 2010; 90: 398-410

13. Chen R. Interactions between inhibitory and excitatory circuits in the human motor cortex. Exp Brain Res 2004; 154: 1-10

14. Vines BW, Nair D, Schlaug G. Contralateral and ipsilateral motor effects after transcranial direct current stuimulation. NeuroReport 2006; 17: 671-674
15. Fregni F, Boggio PS, Mansur CG, Wagner T, Ferreira MJ, Lima MC, Rinonatti SP, Marcolin MA, Freedman SD, Nitsche MA, et al. Transcranial direct current stimulation of the unaffected hemisphere in stroke patients. NeuroReport 2005; 16: 1551-1555

16. Boggio PS, Nunes A, Rigonatti SP, Nitsche MA, Pascual-Leone A, Fregni F. Repeated sessions of noninvasive brain DC stimulation is associated with motor function improvement in stroke patients. Restor Neurol Neurosci 2007; 25: 123-129

17. Nowak DA, Grefkes C, Ameli M, Fink GR. Interhemispheric competition after stroke: brain stimulation to enhance recovery of function of the affected hand. Neurorehabil Neural Repair 2009; 23; 641-656

18. Hackel ME, Wolfe GA, Bang SM, Canfield JS. Changes in hand function in the aging adult as determined by the Jebsen Test of Hand Function. Phys Ther 1992; 72: 373-377

19. Kim YH, Choi MS, Kim BO. Assessment of hand function in normal Korean Adults by Jebsen hand function test. J Korean Acad Rehab Med 1984; 8: 5

20. Hummel FC, Heise K, Celink P, Floel A, Gerloff C, Cohen LG. Fascilitation skilled right hand motor function in older subjects by anodal polarization over the left primary motor cortex. Neurobilology of Aging 2010; 31: 2160-2168

21.Jang SH, Ahn SH, Byun WM, Kim CS, Lee MY, Kwon YH. The effect of transcranial direct current stimulation on the cortical activation by motor task in the human brain: an fMRI study. Neurosci Lett 2009; 460: $117-120$

22. Vines BW, Nair D, Schlaug G. Modulating activity in the motor cortex affects performance for the two hands differently depending upon which hemisphere is stimulated. Eur J Neurosci 2008; 28: 1667-1673 